Acta Technologica Agriculturae 4

Nitra, Slovaca Universitas Agriculturae Nitriae, 2016, pp. 101-106

\title{
STUDY ON NONLINEAR MODEL OF DYNAMICS OF A SYSTEM 'EXTRUDER ELASTIC AUGER WORKING BODY'
}

\author{
Oleg LYASHUK' ${ }^{1}$, Mariya SOKIL ${ }^{2}$, Volodymyr KLENDIY ${ }^{1}$, Olena SKYBA ${ }^{1}$, Victor DMYTRENKO 3 \\ ${ }^{1}$ Ternopil Ivan Pul'uj National Technical University, Ukraine \\ ${ }^{2}$ Lviv Polytechnic National University, Ukraine \\ ${ }^{3}$ Vinnytsia National Agrarian University, Ukraine
}

The model of dynamics of a system 'extruder elastic auger working body' is developed. The design of an extruder elastic screw working body is worked out. The article provides the proposition of calculation technique for determining of the theoretical dependences that would analyse the laws of amplitude changing and oscillation phase of a system 'elastic auger working body' in the non-resonant case. It also provides analytical dependences determining the characteristic features of different geometrical parameters of an extruder working body.

Keywords: extruders; wafers; feed grain processing; bulky loads; dynamic modelling

Nowadays, one of the most important tasks of the national economy of Ukraine is to develop new competitive equipment for manufacturing of cattle feed that would be efficiently mixed. Therefore, the challenge is to study the dynamics of extruder design while processing the feed grain with a mixture by-products from grain production and minerals.

The quality of a final product, especially the density of packaged components of a wafer, its homogeneity, structure, etc., largely depends on the dynamics of motion of bulky loads (wheat, peas, corns) in the working area of an auger machine. Therefore, to form the bulky loads into wafers, it is necessary to study the dynamics of motion of an extruder working body. The problems of moving of bulky loads along the different surfaces were solved on the basis of several different principles, from the material points system motion along the surface (Vasylenko, 1960; Blehman and Dzhanelidze, 1964; Ganiev and Ukrainskyi, 1975) to continuum (Stots'ko et al., 2002; Stots'ko et al., 2001; Subach, 1991; Subach, 1996) of null (similar to liquid) or finite stiffness (similar elastic body). Therefore, the dynamic processes in bulky loads are insufficiently studied. Firstly, a large number of differential equations of motion (usually nonlinear) should be analysed. Secondly, it is difficult to generalise the choice of hypotheses of interaction between individual points of the system due to individual properties of bulky loads. The modern scientists (Stots'ko et al., 2002; Stots'ko et al., 2001; Subach, 1991; Subach, 1996) carried out the observations of the continuum, based on its integral indicators. Such consideration of bulky loads allowed the developing of mathematical models of dynamics of working body motion in bulky loads. However, the qualitatively different processes were noticed during the motion of bulky loads through a modernised auger screw.
The objective is to define the dynamic process and especially to develop dependencies for comprehensive assessment of the entire spectrum of forces, system parameters and boundary conditions.

\section{Material and methods}

In order to define the dynamics of the system under study, the general principles of developing of mathematical models of the dynamics of mechanical systems should be applied (Andronov et al., 1981; Babakov, 1965).

The periodic disturbances influence the right end of an elastic auger screw (Figure 1). Therefore, to study the dynamics of a system model, the non-resonant and resonant cases should be considered.

In the proposed system model, the displacement of bulky loads is defined by means of an elastic working body. In order to calculate the mathematical model, it is necessary to analyse the motion of continuum along the entire working area. Thus, the one-dimensional elastic body, along which the continuum travels, should be considered as an analytical model. For this body, the centre of gravity movement of a section with the coordinate $x$ in random time $t$ is clearly defined by the function $u(x, t)$. Therefore, the force of inertia of a specified element equals $d m \frac{d^{2} u}{d t^{2}}$, where $d m$ represents the mass of a specified element. The mass is equal to the sum of the masses of continuum $d m_{1}$ and the elastic body $d m_{2}$, that is $d m=d m_{1}+d m_{2}$.

Therefore, $d m_{1}=\rho_{1}(x) d x, d m_{2}=\rho_{2}(x) d x, \rho_{1}(x)$ and $\rho_{2}(x)$ represent the volume weight referring to continuum and the elastic body. Elastic properties of the auger screw model under study are depicted by applying the quasi-linear 


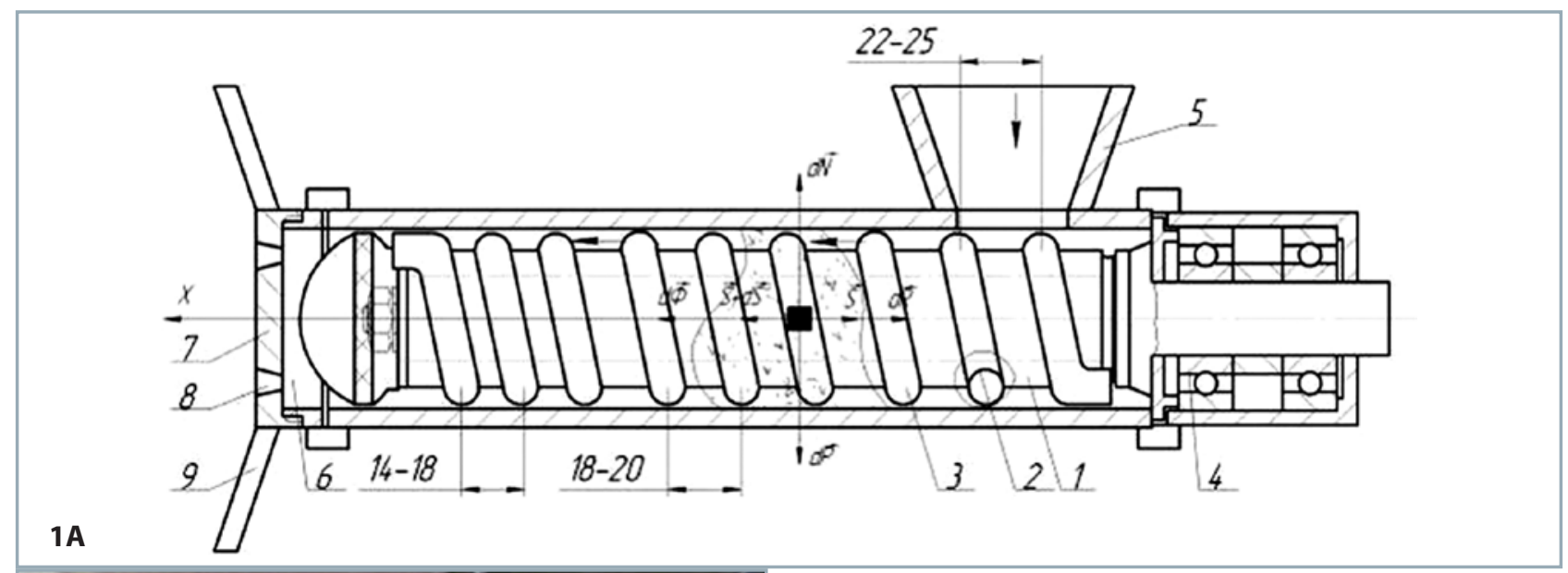

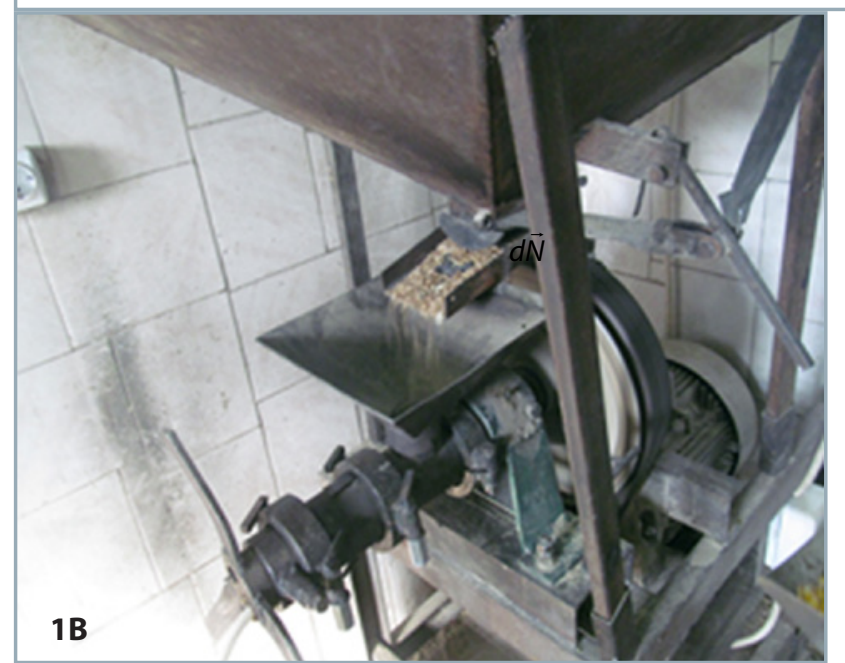

Figure 1A-B Analytical model 'extruder elastic auger working body'

1 - shaft; 2 - spiral groove; 3 - coil spring; 4 - cylindrical body; 5 - loading hopper; 6 - unloading zone; 7 nut; 8 - calibration holes; 9 - handle, $d \vec{P}$ - weight of a selected element; $d \vec{N}$ - normal reaction; $d \vec{R}$ resistance; $d \vec{\Phi}$ - inertia of relative motion of selected element with a screw spiral; $\vec{S}$ - force acting on the right end of a selected element from the side of the cut off part of elastic screw; $\vec{S}+d \vec{S}$ - force acting on the left end of a selected element from the side of the cut off part of elastic screw

law of elasticity $\sigma=E \varepsilon+\mu f(\varepsilon, \dot{\varepsilon})$, where $\sigma$ represents the normal tension in a cross-section of the system model, $\varepsilon$ and $\dot{\varepsilon}$ represent the relative deformation and its rate; $\mu f(\varepsilon$, $\dot{\varepsilon})$ represents a feature that indicates the deviation of elastic model material properties from the linear law; $\mu$ represent a small parameter indicating a fractional deviation from the specified properties of the linear law. In this case, the values of internal forces $S$ and $S+d S$ can be written as:

$$
\begin{gathered}
S=E A \frac{\partial u(x, t)}{\partial x}+A \mu f\left(\frac{\partial u(x, t)}{\partial x}, \frac{\partial^{2} u(x, t)}{\partial x \partial t}\right) \\
S+d S=E A \frac{\partial u(x, t)}{\partial x}+A \mu f\left(\frac{\partial u(x, t)}{\partial x}, \frac{\partial^{2} u(x, t)}{\partial x \partial t}\right)+ \\
+\frac{\partial}{\partial x}\left[E A \frac{\partial u(x, t)}{\partial x}+A \mu f\left(\frac{\partial u(x, t)}{\partial x}, \frac{\partial^{2} u(x, t)}{\partial x \partial t}\right)\right] d x
\end{gathered}
$$

The relative deformation for a one-dimensional model of the elastic body is determined by the dependence $\varepsilon=\frac{\partial u(x, t)}{\partial x}$, where $A$ is the area of its cross section. This statement is considered in the above correlations. In order to pass the vector of correlation into its scalar form, it is necessary to consider the kinetostatics equation for the specified element:

$$
\begin{gathered}
\left(\rho_{1}(x)+\rho_{2}(x)\right) \frac{d^{2} u(x, t)}{d t^{2}}-\frac{\partial}{\partial x}\left[E A \frac{\partial u(x, t)}{\partial x}+A \mu f\left(\frac{\partial u(x, t)}{\partial x}, \frac{\partial^{2} u(x, t)}{\partial x \partial t}\right)\right]+ \\
+R\left(\frac{d u(x, t)}{d t}\right)=0
\end{gathered}
$$

However, as the continuum travels with the relative velocity $V$ along the elastic body of specified element, the inertial force of continuum can be represented by the equation:

$$
\rho_{2}(x) \frac{d^{2} u(x, t)}{d t^{2}}=
$$

$\rho_{2}(x)\left[\frac{\partial^{2} u(x, t)}{\partial t^{2}}+2 \frac{\partial^{2} u(x, t)}{\partial x \partial t} V+\frac{\partial^{2} u(x, t)}{\partial x^{2}} V^{2}+\frac{\partial u(x, t)}{\partial x} \frac{d V}{d t}\right]$

Thus, the differential equation of the dynamics of a system 'continuum - elastic auger screw' can be written as:

$$
\begin{gathered}
\frac{\partial^{2} u(x, t)}{\partial^{2} t}-\frac{1}{\rho_{1}(x)+\rho_{2}(x)} \\
\left\{\frac{\partial}{\partial x}\left[E A \frac{\partial u(x, t)}{\partial x}+A \mu f\left(\frac{\partial u(x, t)}{\partial x}, \frac{\partial^{2} u(x, t)}{\partial x \partial t}\right)\right]+R\left(\frac{d u(x, t)}{d t}\right)-\right. \\
\left.-\rho_{2}(x)\left[\frac{\partial^{2} u(x, t)}{\partial t^{2}}+2 \frac{\partial^{2} u(x, t)}{\partial x \partial t} V+\frac{\partial^{2} u(x, t)}{\partial x^{2}} V^{2}+\frac{\partial u(x, t)}{\partial x} \frac{d V}{d t}\right]\right\}=0
\end{gathered}
$$

To improve the structure and uniformity of continuum, the right end of the elastic spiral auger screw undergoes the periodic disturbance of motion, in particular, it performs harmonic oscillations. To present them in the mathematical model of the process, it is necessary to define the boundary conditions for the Equation 5. The periodic motion of the right end should be written using boundary conditions:

$$
u(x, t)_{\left.\right|_{x=0}}=h \sin \theta, \theta=\omega t+\varphi
$$


where:

$h, \omega, \varphi$ - are the steels. Similarly, the boundary condition for the left end is written taking into account the fact, that it is fixed:

$$
u(x, t)_{\mid x=1}=0
$$

\section{Results and discussion}

The formula $\Omega \neq \frac{r}{s} \omega$ is valid for the non-resonant cases; the formula $\Omega \approx-\frac{r}{s} \omega(r, s$ are relatively prime numbers) must be applied to resonant cases. Based on the above-mentioned analytical model, a simple non-resonant case should be considered at first. Nonlinear forces and boundary conditions cause not only changes in the time of the main parameters of wave processes (amplitude $a$ and phase $\varphi)$, but also distortion, to a certain extent, in the shape of the waves. Thus, according to the basic idea of asymptotic methods of nonlinear mechanics, the non-resonant solution of disturbance problems allows to represent a mathematical model of the working body dynamics in continuum:

$$
\begin{gathered}
\frac{\partial^{2} u(x, t)}{\partial^{2} t}+\frac{\rho_{20}}{\rho_{10}+\rho_{20}} \frac{\partial^{2} u(x, t)}{\partial x \partial t}-\frac{\left(E A-\rho_{20}\right)}{\rho_{10}+\rho_{20}} \frac{\partial^{2} u(x, t)}{\partial x^{2}}= \\
=\mu f_{1}\left(x, \frac{\partial u(x, t)}{\partial t}, \frac{\partial u(x, t)}{\partial x} \frac{\partial^{2} u(x, t)}{\partial t \partial x}\right) \\
u(x, t)_{\mid x=0}=\mu h_{0} \sin \theta \\
u(x, t)_{\mid x=1}=0
\end{gathered}
$$

where:

$$
\begin{aligned}
& f_{1}\left(x \frac{\partial u(x, t)}{\partial t}, \frac{\partial u(x, t)}{\partial x} \frac{\partial^{2} u(x, t)}{\partial t \partial x}\right)=\frac{1}{\rho_{10}+\rho_{20}} \\
& \left\{-\rho_{21}(x)\left[\frac{\partial^{2} u(x, t)}{\partial x \partial t}-\frac{\left(E A-\rho_{20}\right)}{\rho_{10}+\rho_{20}} \frac{\partial^{2} u(x, t)}{\partial x^{2}}\right]+\right. \\
& +A \frac{\partial}{\partial x} f\left(\frac{\partial u(x, t)}{\partial x}, \frac{\partial^{2} u(x, t)}{\partial x \partial t}\right)-R\left(\frac{\partial u(x, t)}{\partial t}\right)- \\
& \left.-\rho_{21}(x)\left[2 \frac{\partial^{2} u(x, t)}{\partial x \partial t} V+\frac{\partial^{2} u(x, t)}{\partial x^{2}} V^{2}+\frac{\partial u(x, t)}{\partial x} \frac{d V}{d t}\right]\right\}
\end{aligned}
$$

The left side of the above differential equation is linear with additional constant coefficients; the right sides of the equation (Equation 8) and boundary conditions (Equation 9) are proportional to a small parameter. Therefore, in order to develop the solution of the given problem, the general ideas of approximate analytical methods of disturbances can be applied (Stots'ko, 2002) in the first consideration of asymptotic series:

$$
\begin{aligned}
u(x, t)= & a(\cos (K x+\Omega t+\varphi)-\cos (X x-\Omega t-\varphi))+ \\
& +\varepsilon U(a, x, \phi, \theta), \phi=\Omega t+\varphi
\end{aligned}
$$

where:

the function $U(a, x, \phi, \theta)$ should be $2 \pi$ that is alternating in phases of the own and forced oscillations, that is, $\phi, \theta$, and should meet the boundary conditions arising from Equation 9:

$$
\begin{gathered}
U(a, x, \phi, \theta)_{\left.\right|_{x=0}}=h_{0} \sin \theta \\
U(a, x, \phi, \theta)_{\left.\right|_{x=0}}=0
\end{gathered}
$$

In addition, the changes in the time of amplitude and phase of the wave process are caused by the resistance and non-uniformity of mass distribution along the working body (auger screw), and by the variable speed of bulky loads motion in relation to an elastic helical spiral. The influence of the above factors on the laws of changes in the time of amplitude and phase of the wave process is analysed below.

Unidentified laws of changing the parameters $a$ and $\phi$ are studied in the correlations:

$$
\frac{d a}{d t}=\mu A_{1}(a) \quad \frac{d \phi}{d t}=\Omega+\mu b_{1}(a)
$$

where:

the unknown right parts $A_{1}(a)$ and $B_{1}(a)$ are located in such a way that the asymptotic representation (Equation 10) with an accuracy to the size of order satisfies the Equation 8 with the boundary conditions described in Equation 9

By means of differentiation of the Dependence 10 by the variables $t$ and $x$, the following formula is deduced:

$$
\begin{gathered}
u_{u}(x, t)=-a \Omega^{2}(\cos (\mathrm{K} x+\phi)-\cos (\mathrm{H} x+\phi))+2 \Omega \frac{d a}{d t} \\
(\sin (\mathrm{K} x+\phi)+\sin (\mathrm{H} x-\phi))-\left(\frac{d^{2} a}{d t^{2}}+2 \Omega a \frac{d \phi}{d t}+a\left(\frac{d \phi}{d t}\right)^{2}\right) \\
(\cos (\mathrm{K} x+\phi)-\cos (\mathrm{H} x+\phi))+\mu\left\{\frac{\partial U_{1}(a, x, \phi, \theta)}{\partial a} \frac{d^{2} a}{d t^{2}}+\right. \\
\left.+\frac{\partial U_{1}(a, x, \phi, \theta)}{\partial \phi} \frac{d^{2} \phi}{d t^{2}}+\omega^{2} \frac{\partial^{2} U_{1}(a, x, \phi, \theta)}{\partial \theta^{2}}\right\}+ \\
+\mu\left\{\frac{\partial^{2} U_{1}(a, x, \phi)}{\partial a^{2}}\left(\frac{d a}{d t}\right)^{2}+\frac{\partial^{2} U_{1}(a, x, \phi)}{\partial \phi^{2}}\left(\frac{d \phi}{d t}\right)^{2}+\right. \\
\left.+\frac{\partial^{2} U_{1}(a, x, \phi)}{\partial a \partial \phi} \frac{d a}{d t} \frac{d \phi}{d t}+\mu^{2}\right\} \\
u_{x x}(x, t)=-a\left(\mathrm{~K}^{2} \cos (\mathrm{K} x+\phi)-\mathrm{H}^{2} \cos (\mathrm{H} x-\phi)\right)+ \\
+\mu \frac{\partial^{2} U_{1}(a, x, \phi)}{\partial x^{2}}+\mu^{2} \ldots \\
u_{t x}(x, t)=-a \Omega(\mathrm{K} \cos (\mathrm{K} x+\phi)+\mathrm{H} \cos (\mathrm{H} x-\phi))-a \frac{d \varphi}{d t}
\end{gathered}
$$

$(\mathrm{K} \cos (\mathrm{K} x+\phi)+\mathrm{H} \cos (\mathrm{H} x-\varphi))-\frac{d a}{d t}(\mathrm{~K} \sin (\mathrm{K} x+\phi)-\mathrm{H} \sin (\mathrm{H} x-\phi))+$

$$
\mu\left\{\frac{\partial^{2} U_{1}(a, x, \phi)}{\partial x \partial a} \frac{d a}{d t}+\frac{\partial^{2} U_{1}(a, x, \phi)}{\partial x \partial \phi} \frac{d \phi}{d t}+\omega \frac{\partial^{2} U_{1}(a, x, \phi, \theta)}{\partial x \partial \theta}\right\}+\mu_{\ldots}^{2}
$$

If we insert in the initial equation with the abovementioned values instead of the function $u(x, t)$ and its derivatives, we will get the linear differential equation that links the unknown parameters $\dot{a} \dot{\phi}$ and the function $U_{1}(a$, $x, \phi, \theta)$ : 


$$
\begin{gathered}
L\left(U_{1}(a, x, \phi, \theta)\right)=\Omega^{2} \frac{\partial^{2} U_{1}(a, x, \phi, \theta)}{\partial \phi^{2}}+\omega^{2} \frac{\partial^{2} U_{1}(a, x, \phi, \theta)}{\partial \theta^{2}}+ \\
+\frac{1}{\rho_{10}+\rho_{20}} \times\left(2 V \rho_{20} \Omega \frac{\partial^{2} U_{1}(a, x, \phi, \theta)}{\partial x \partial \phi}+2 V \rho_{20} \omega \frac{\partial^{2} U_{1}(a, x, \phi, \theta)}{\partial x \partial \phi}-\right. \\
\left.-\left(A E-\rho_{20} V^{2}\right) \frac{\partial^{2} U_{1}(a, x, \phi, \theta)}{\partial x^{2}}\right)= \\
=\bar{f}_{1}(a, x, \phi)-2\left[\alpha_{1} \sin (\mathrm{K} x+\phi)+\alpha_{2} \sin (\mathrm{H} x-\phi)\right] \frac{d a}{d t}- \\
-2 a\left[\alpha_{1} \cos (\mathrm{K} x+\phi)-\alpha_{2} \cos (\mathrm{H} x-\phi)\right] \frac{d \phi}{d t}
\end{gathered}
$$

where:

$$
\begin{aligned}
& \alpha_{1}=\Omega+\frac{2 \rho_{20} V K}{\rho_{10}+\rho_{20}}, \alpha_{2}=\Omega-\frac{2 \rho_{20} V H}{\rho_{10}+\rho_{20}} \\
& \bar{f}_{1}(a, x, \phi)=f_{1}\left(x, \frac{\partial u(x, t)}{\partial t}, \frac{\partial u(x, t)}{\partial x} \frac{\partial^{2} u(x, t)}{\partial t \partial x}\right)_{\substack{u=a(\cos (K x+\phi)-\cos (H x-\phi)), u_{x}=-a(K \sin (x x+\phi)-H \sin (x-\phi)), u_{t}=-a \Omega(\sin (K x+\phi)+\sin (H x-\phi))}}
\end{aligned}
$$

In addition, in this equation, the function $U(a, x, \phi, \theta)$ must meet the boundary conditions described by the Equation 11. Therefore, the resulting solution of Equation 7 can be found (Stots'ko et al., 2001) in the following set of functions:

$$
U(a, x, \phi, \theta)=\bar{U}_{1}(a, x, \phi, \theta)+\tilde{U}_{1}(a, x, \phi, \theta)
$$

Let us consider the function $\bar{U}_{1}(a, x, \phi, \theta)$ as a solution of differential equation:

$$
\frac{\partial^{2} \bar{U}_{1}(a, x, \phi, \theta)}{\partial x^{2}}=0
$$

This function satisfies the boundary conditions:

$$
\begin{gathered}
\bar{U}_{1}(a, x, \phi, \theta)_{\mid x=0}=h_{0} \sin \theta \\
\bar{U}_{1}(a, x, \phi, \theta)_{\mid x=1}=0
\end{gathered}
$$

Then, as results from Functions 15, 16 and 17, the function $\widetilde{U}_{1}(a, x, \phi, \theta)$ is a solution of the differential equation:

$$
\begin{gathered}
L\left(\tilde{U}_{1}(a, x, \phi, \theta)\right)=-L\left(\bar{U}_{1}(a, x, \phi, \theta)\right)+\bar{f}_{1}(a, x, \phi)- \\
-2\left[\alpha_{1} \sin (\mathrm{K} x+\phi)+\alpha_{2} \sin (\mathrm{H} x-\phi)\right] \frac{d a}{d t}- \\
-2 a\left[\alpha_{1} \cos (\mathrm{K} x+\phi)+\alpha_{2} \cos (\mathrm{H} x-\phi)\right] \frac{d \phi}{d t}
\end{gathered}
$$

and it should meet the boundary conditions:

$$
\tilde{U}_{1}(a, x, \phi, \theta)_{\mid x=0}=0, \quad \tilde{U}_{1}(a, x, \phi, \theta)_{\mid x=1}=0
$$

Based on Functions 16 and 17, the function $\bar{U}_{1}(a, x, \phi, \theta)$ can be easily derived:

$$
\bar{U}_{1}(a, x, \phi, \theta)=\frac{h_{0}}{l}(I-x) \sin \theta
$$

This allows the developing of the right side of the differential Dependence Equation 18:

$$
\begin{gathered}
\tilde{f}(a, x, \phi, \theta)=\omega^{2} \frac{h_{0}}{l}(I-x) \sin \theta+\frac{2 V \rho_{20}}{\rho_{10}+\rho_{20}} \frac{h_{0}}{l} \omega \cos \theta+ \\
+\bar{f}_{1}(a, x, \phi)-2\left[\alpha_{1} \sin (\mathrm{K} x+\phi)+\alpha_{2} \sin (\mathrm{H} x-\phi)\right] A_{1}(a)- \\
-2 a\left[\alpha_{1} \cos (\mathrm{K} x+\phi)-\alpha_{2} \cos (\mathrm{H} x-\phi)\right] B_{1}(a)
\end{gathered}
$$

In order to determine the unknown functions $\dot{a}(t)$ and $\dot{\phi}(t)$ from the differential Dependence Equation 18, it is necessary to determine the additional conditions of the function $\tilde{U}_{1}(a, x, \phi, \theta)$. It means that the function should not expand in Fourier series of the first modes $\phi$ :

$$
\int_{0}^{2 \pi} \widetilde{U}_{1}(a, x, \phi, \theta)\left\{\begin{array}{c}
\cos \phi \\
\sin \phi
\end{array}\right\} d \phi=0
$$

The physical meaning of dependencies is as follows: the amplitude of oscillatory process of a system 'continuum elastic auger screw' coincides with its first mode. Simultaneously, the conditions described by Function 22 allow obtaining of correlations, which define the laws changing the amplitude and oscillation phase of the object under study in the non-resonant case.

$$
\frac{d a}{d t}=
$$

$$
\begin{aligned}
& =\varepsilon \frac{\int_{0}^{12 \pi} \int_{0}^{2 \pi} \bar{f}_{1}(a, x, \phi)\left[\alpha_{1}\left(\sin \kappa x+\alpha_{2} \sin \chi x\right) \sin \phi+\left(\alpha_{1} \cos \kappa x-\alpha_{2} \cos \chi x\right) \cos \phi\right] d \phi d x}{2 \pi /\left(\alpha_{1}^{2}+\alpha_{2}^{2}\right)} \\
& \frac{d \phi}{d t}=\Omega+
\end{aligned}
$$

$+\varepsilon \frac{\int_{0}^{1} \int_{0}^{2 \pi} \bar{f}_{1}(a, x, \phi)\left[\left(\alpha_{1} \sin \kappa x+\alpha_{2} \sin \chi x\right) \sin \phi-\left(\alpha_{1} \cos \kappa x-\alpha_{2} \cos \chi x\right) \cos \phi\right] d \phi d x}{2 \pi /\left(\alpha_{1}^{2}+\alpha_{2}^{2}\right)}$

In the given differential equation, the properties of its right part are considered in the Dependence Equation 18:

$$
\begin{gathered}
\int_{0}^{2 \pi} L\left(\bar{U}_{1}(a, x, \phi, \theta)\right)\left\{\begin{array}{c}
\cos \phi \\
\sin \phi
\end{array}\right\} d \phi= \\
\int_{0}^{2 \pi}\left[\omega_{2} \frac{h_{0}}{I}(I-x) \sin \theta+\frac{2 V \rho_{20}}{\rho_{10}+\rho_{20}} \frac{h_{0}}{l} \omega \cos \theta\right]\left\{\begin{array}{c}
\cos \phi \\
\sin \phi
\end{array}\right\} d \phi=0
\end{gathered}
$$

According to the dependencies described by Function 16, in the first non-resonant case, the small periodic disturbances do not influence the laws changing the amplitude and frequency of the dynamic process. The laws explaining the deviation of elastic properties of an auger screw by means of the linear law of external and internal friction determine this process. The influence of these factors can be observed in the dependence of natural oscillations frequency on the amplitude and rate in time. The influence of periodic disturbance in the non-resonant cases can be observed in changing the wave form; the periodic disturbance is defined by functions $\bar{U}_{1}(a, x, \psi, \theta)$ and $\widetilde{U}_{1}(a, x, \psi, \theta)$. The first function is known (see Dependence Equation 18); in accordance to Equations 16, 17 and 20, the second function can be written as:

$$
\tilde{U}_{1}(a, x, \psi, \theta)=\sum_{m \neq 1} \sum_{n} \sum_{n} \tilde{U}_{m n j}(a) \exp i(m \phi+j \theta) X_{n}(x)
$$


where:

$\left\{X_{n}(x)\right\}$ represents a complete system of functions that satisfies boundary conditions described by the Equation 17. Such system of functions can be represented as $\left\{X_{n}(x)\right\}=$ $=\left\{\sin \frac{n \pi}{l} x\right\}$. It is a rule that the first modes of oscillation mainly influence the dynamic process. Therefore, in the Representation 25, it is sufficient to apply only some of the first members of the function expansion. It is not difficult to solve this problem. In particular, if we consider only the first members of the function expansion, we will get:

$$
\begin{gathered}
U_{00 s}(a)=\omega^{2} h_{0}, \quad U_{01 c}(a)=0, \quad U_{0 c}(a)=0 \\
U_{01 s}(a)=\frac{\omega^{2} h_{0}}{I}\left(\omega^{2}-\frac{A E-\rho_{20} V^{2}}{\rho_{10}+\rho_{20}}\left(\frac{\pi}{I}\right)^{2}\right)^{-1} D_{1} \\
\left\{4 \Omega^{2} \frac{A E-\rho_{20} V^{2}}{\rho_{10}+\rho_{20}}\left(\frac{\pi}{I}\right)^{2}\right\} U_{210}(a)- \\
-4 V \Omega \frac{\rho_{20} i}{\rho_{10}+\rho_{20}} \frac{4 \pi}{3 l} U_{210}(a)=-F_{210}(a)
\end{gathered}
$$

where:

$D_{n}$ is the function expansion coefficients, $g(x)$ represents the specified system of functions. The above system of linear equations determines the unknown coefficients $U_{00 c}(a), \ldots$, $U_{210}(a)$.

To define the main parameters of screw extruders, it is necessary to consider the peculiarities of their designs, the technology of production and the performance. In order to determine the volumetric productivity of elastic screw extruder, it is necessary to define the area of passage section and the coefficients determined experimentally for different types of grain materials.

Based on the model of dynamic system, the analytical dependences are developed and the semi graphical dependence $Q=f(D, h)$ is deduced for the determination of the performance.

The method for determination of extruder volumetric productivity (Figure 1) depends on the geometrical

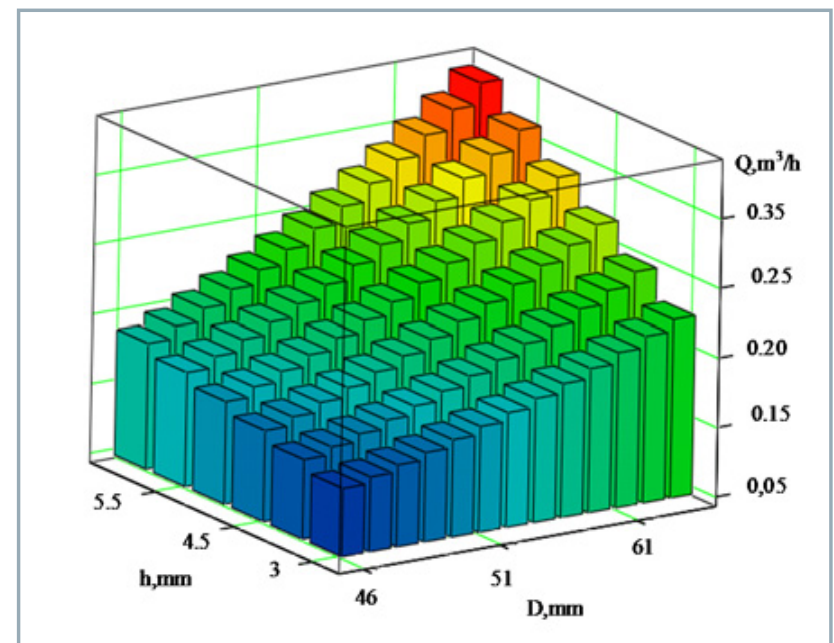

Figure 2 Dependence of the performance on regular geometric parameters of extruder working body

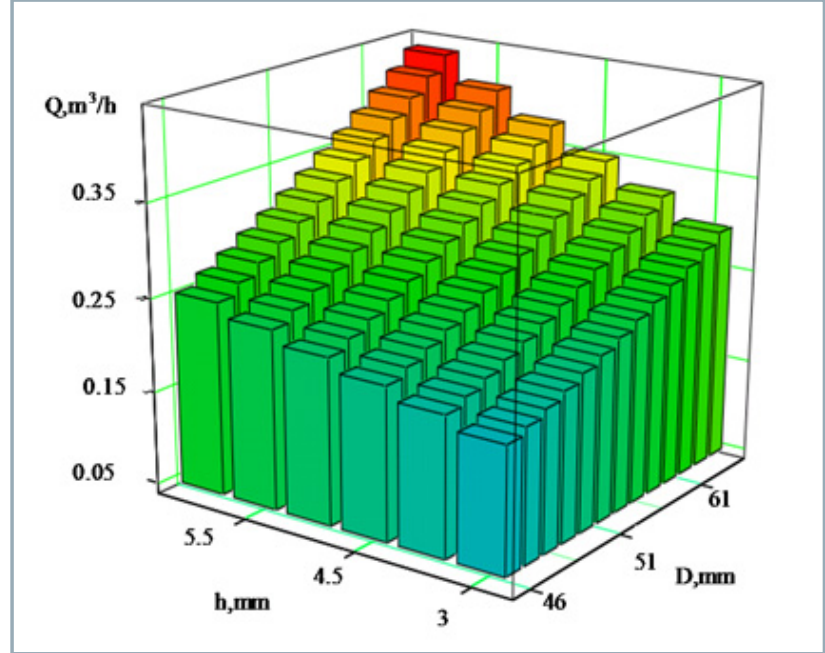

Figure 3 Dependence of the performance on the change of a pitch of extruder working body

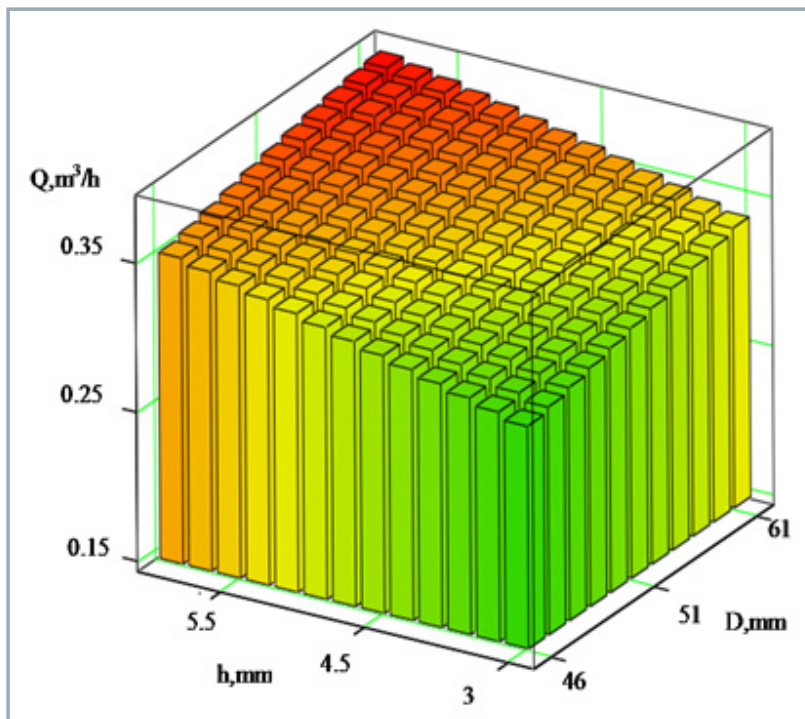

Figure 4 Dependence of the performance on the change of the screw channel depth of extruder working body

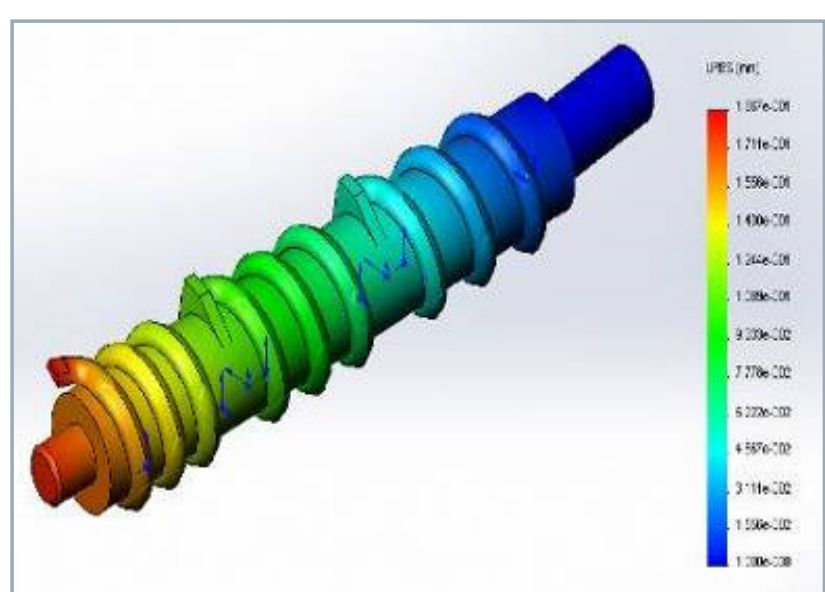

Figure 5 Distribution of equivalent stresses along the surface of the working body 


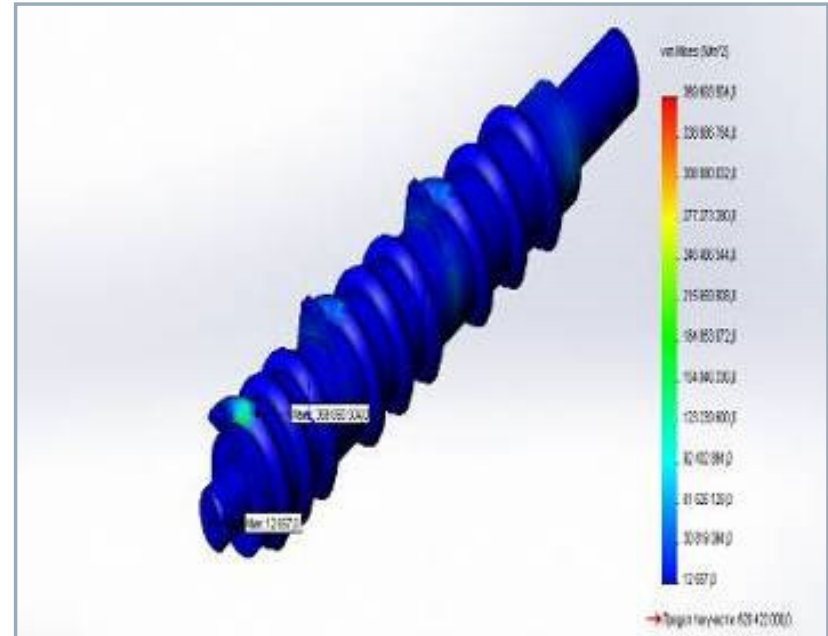

Figure 6 Distribution of equivalent placements along the surface of the working body

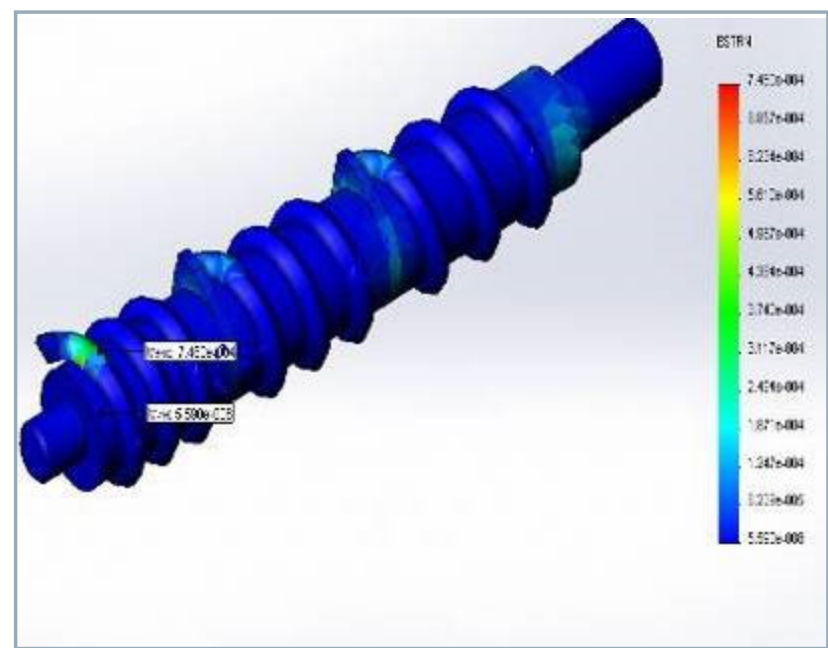

Figure 7 Distribution of equivalent deformations along the surface of the working body

parameters of the working body. This method is based on the calculation of stiffness and strength parameters of working body design. The working body was made of compound steel 40X with strength limits of 620MPa (Vasylenko, 1960 Stots'ko et al., 2001). The calculations showed that the stress arising during the transportation of the continuum reaches $370 \mathrm{MPa}$ (Figures 5-7). The Figure 7 shows that if the corresponding loads are applied to the extruder working body, the maximum deformations occur at the end of an auger spiral and they are within $7.4510^{-4}$, as the last turn of an auger is the most loaded.

\section{Conclusions}

According to theoretical results, the graphical dependences present the technique of developing the approximate analytical solution of the dynamic model of a system 'extruder elastic auger working body'. The technique is based on:

- the main provisions of the wave theory of motion;

- the principles of single-frequency oscillations in non-linear mechanical systems;

- the application of the main ideas of asymptotic methods of non-linear mechanics to the mechanical systems under study.

The developed technique is based on mathematical modelling. This technique allows determining of the operational load of elastic working body by means of the main technological parameters and rheological properties of the continuum

\section{References}

VASYLENKO, P. M. 1960. The theory of motion of a particle along a rough surface of agricultural machinery. Kyiv : Academy of Agricultural Sciences of USSR, 1960, 283 pp.

ANDRONOV, A. A. - VITT, A. P. - KHAYKIN, S. E. 1981. Theory of oscillations. Moscow : Nauka, 1981, 568 pp.

BABAKOV, I. M. 1965. Theory of oscillations. Moscow : Nauka, 1965 $560 \mathrm{pp}$

BLEHMAN, I. I. - DZHANELIDZE, G.Y. 1964. Vibration displacement. Moscow : Nauka, 1964, 410 pp.

GANIEV, R. F. - UKRAINSKYI L. E. 1975. Dynamics of particles when subjected to vibration. Kyiv : Naukova dumka, 1975, $168 \mathrm{pp}$.

STOTS'KO, Z. A. - SOKIL, B. I. - TOPIL'NYTSKYI, V. G. 2002. The impact of structural and kinematic parameters of vibration on the increase of bulk processing intensity. In Ukrainian scientific-technical journal The vibrations in engineering and technology, vol. 4, 2002, no. 25, pp. 46-52.

STOTS'KO, Z. A. - SOKIL, B. I. - TOPIL'NYTSKYI, V. G. 2001. Non-linear motion model of a layer of container working environment of vibration machine of bulk processing the products with varying non-linear parameter. In Mechanical Engineering, vol. 1, 2001, pp. 19-23.

SUBACH, A. P. 1991. Dynamics of machines and processes of bulk vibration and centrifuging process of bulk details. Riga : Znanie, 1991, $400 \mathrm{pp}$.

SUBACH, A. P. 1996. Forced vibrations of the vibro-impact system in an inelastic collision of the masses. On a problem of dynamics. Riga : Znanie, 1996, pp. 67-78. 